\title{
A Rare Case of Acute Cerebrovascular Accident in the Post-Partum Period after Primary Angioplasty during Pregnancy
}

\author{
Ruchi Gupta \\ Soni Manipal Hospital, Jaipur, India \\ Email: ruchi.gupta98@yahoo.com
}

Received 7 November 2015; accepted 12 December 2015; published 15 December 2015

Copyright (C) 2015 by author and Scientific Research Publishing Inc.

This work is licensed under the Creative Commons Attribution International License (CC BY). http://creativecommons.org/licenses/by/4.0/

c) (i) Open Access

\begin{abstract}
Pregnancy-related acute myocardial infarction is rare, but a serious event. We report a case of acute myocardial infarction in a 33-year-old female with 5 months of pregnancy. Angiography, under abdominal shield, revealed $\mathbf{1 0 0 \%}$ thrombotic occlusion in the left anterior descending artery, which was treated successfully with a stent implantation. The patient was found to have hyperhomocysteinemia within the first week of primary percutaneous coronary intervention (PCI). Rest of the follow-up was uneventful with delivery of a healthy baby by elective caesarean section at about 36 weeks of gestation. Within 7 days of post-partum period, the patient displayed signs of cerebrovascular infarction and was managed successfully. To the best of our knowledge, the association between primary PCI during pregnancy and cerebrovascular event in the postpartum period has not been reported previously.
\end{abstract}

\section{Keywords}

Acute Myocardial Infarction, Cerebrovascular Infarction, Hyperhomocysteinemia, Pregnancy, Primary PCI

\section{Introduction}

Myocardial infarction in pregnancy is rare phenomenon, with an estimated frequency of 3 - 10 cases per 0.1 million deliveries [1]. The prognosis is often poor as high maternal mortality and fetal loss is frequently associated with pregnancy-related myocardial infarction [2]. Back in 1988, Cowan et al. reported the first case of percutaneous coronary intervention (PCI) during pregnancy in a 30-year-old woman with myocardial infarction [3]. Since then, numerous cases of coronary angioplasty during pregnancy have been reported. Herein, we present a 
case of acute anterior-wall myocardial infarction developed during the second trimester in a 33-year-old woman in the absence of common risk factors. The patient was treated successfully with percutaneous coronary angioplasty and she delivered a healthy baby after an elective caesarean section. However, a cerebrovascular infarction occurred during the post-partum period. To the best of our knowledge, the association between primary PCI during pregnancy and cerebrovascular event in the postpartum period has not been reported previously.

\section{Case Report}

A 33-year-old female with 5 months of pregnancy presented to our hospital with progressive severe chest pain for more than 20 minutes. She had a strong family history of coronary artery disease. Other coronary risk factors were absent. Analysis of lipid profile displayed slightly elevated levels of total cholesterol (238 mg/dL) and low-density lipoprotein (LDL) cholesterol (154 mg/dL). Other laboratory examinations were unremarkable.

Electrocardiogram of the patient revealed hyperacute $\mathrm{T}$ waves, consistent with an anterior myocardial infarction (Figure 1). A bed-side 2D-echo (Figure 2) verified severe hypokinesia in the LAD territory, with mild mitral regurgitation, and diastolic dysfunction. The left-ventricle ejection fraction was 30\%. In view of symptomatic status, she was transferred emergently for catheterization and possible intervention. Coronary angiography via right femoral artery was performed under abdominal shielding to protect the fetus from radiation. Angiography revealed 100\% thrombotic occlusion in the proximal to mid left anterior descending (LAD) artery (11 mm $\times$ 3.5 mm, Type C lesion; Figure 3). Other coronary vessels were normal. The patient was diagnosed with acute anterior-wall myocardial infarction and single-vessel coronary artery disease with a live intrauterine gestation of 23 weeks 4 days. She was scheduled for primary PCI.

After giving a bolus of heparin, the lesion was crossed with a BMW guidewire (Abbott Vascular, USA) using an EBU 3.5, 6F guiding catheter (Medtronic Inc., USA) through transfemoral route. Then, a $2.5 \times 100 \mathrm{~mm}$ Sprinter balloon (Medtronic Inc., USA) was inflated at 12 atm in the LAD coronary lesion. Subsequently, a $3.5 \times 15 \mathrm{~mm}$ everolimus-eluting stent (Xience Prime; Abbott Vascular, USA) was deployed at 14 atm at the lesion site. During the procedure, eptifibatide bolus and infusion were given as adjunctive medication. The procedure was successful and TIMI grade III flow was achieved in the LAD artery (Figure 4). Post-procedural gynecological consultation was taken and the investigations revealed a normal fetus. Complete coagulation profile was done, which revealed hyper-homocysteinemia. The patient was treated with folic acid $400 \mathrm{mg}$ to achieve normal levels of blood homocysteine. After 2 days of procedure, the patient was discharged in a stable condition with prescription for optimized medical regimen that comprised dual antiplatelet drug therapy with aspirin and clopidogrel. Though treatment with high-dose statin was required, we didn't give it antinataly because of its relative contraindications in pregnancy.

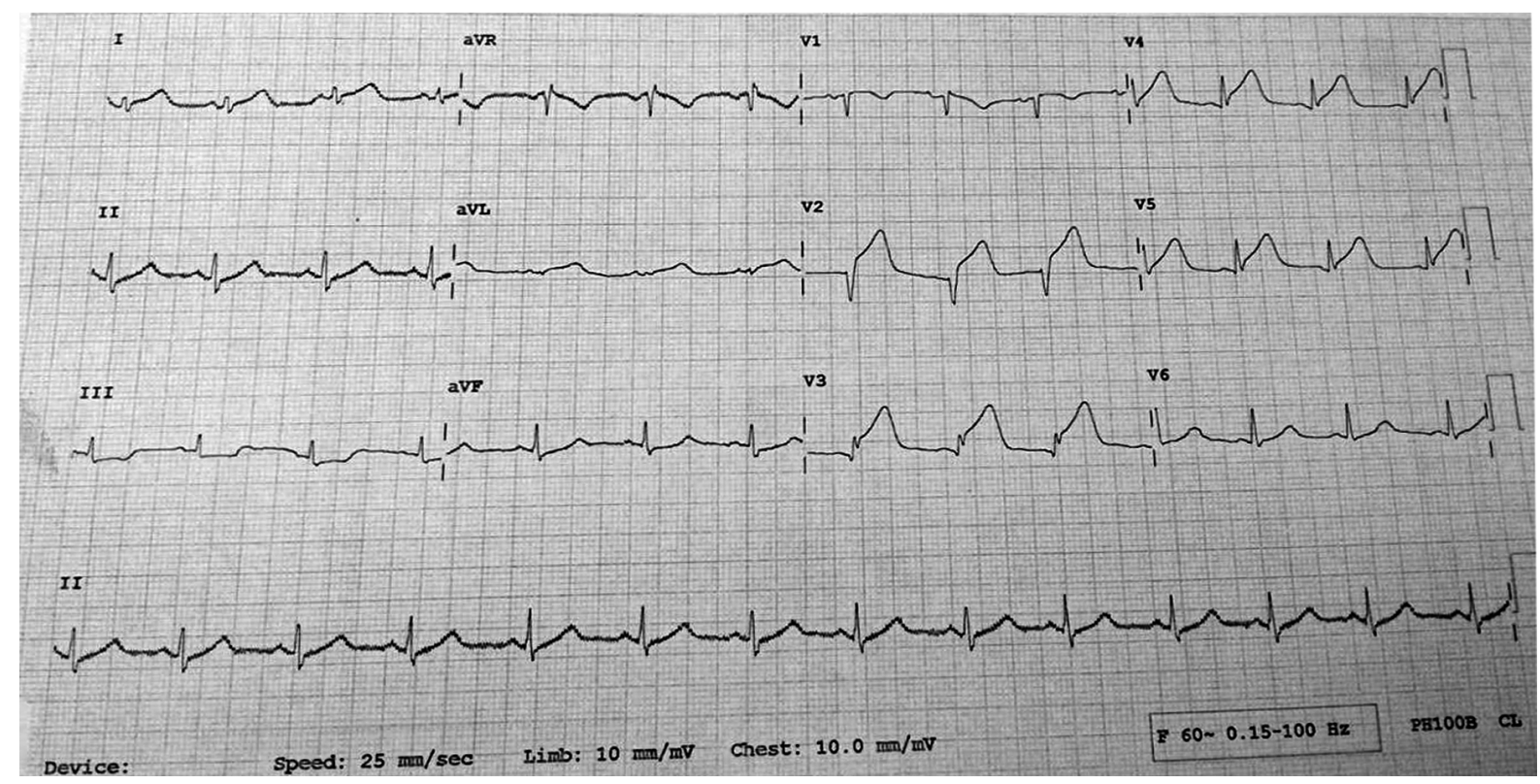

Figure 1. Electrocardiogram of the patient revealed hyperacute T waves and anterior-wall myocardial infarction. 

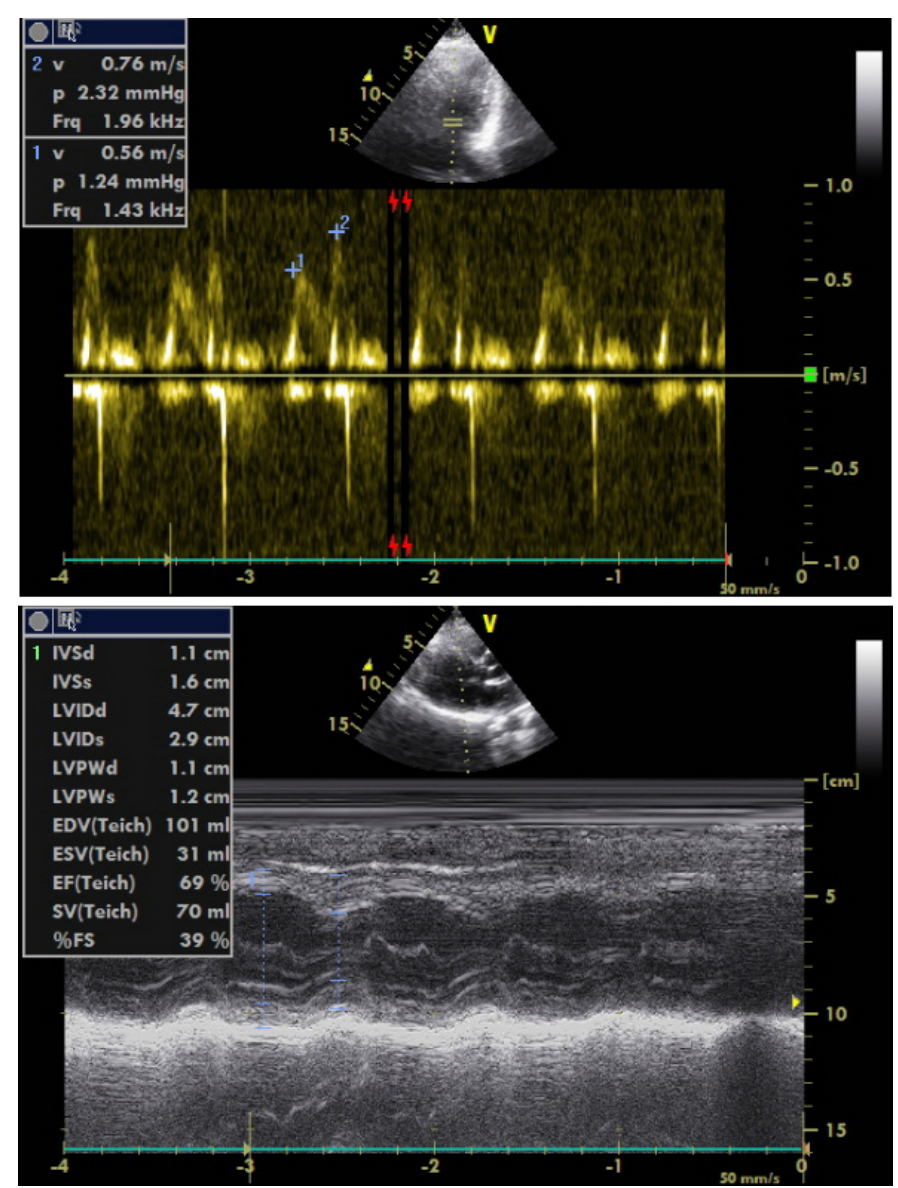

Figure 2. 2D-echo verified severe hypokinesia in the LAD territory.

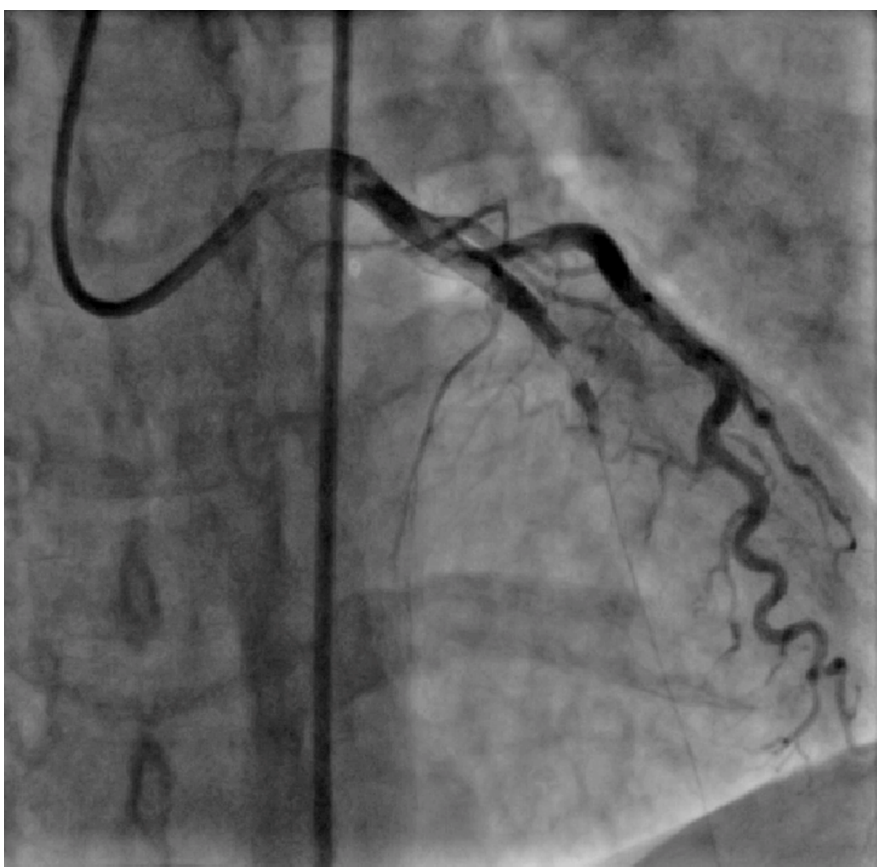

Figure 3. Coronary angiography revealed $100 \%$ thrombotic occlusion in the proximal to mid LAD. 


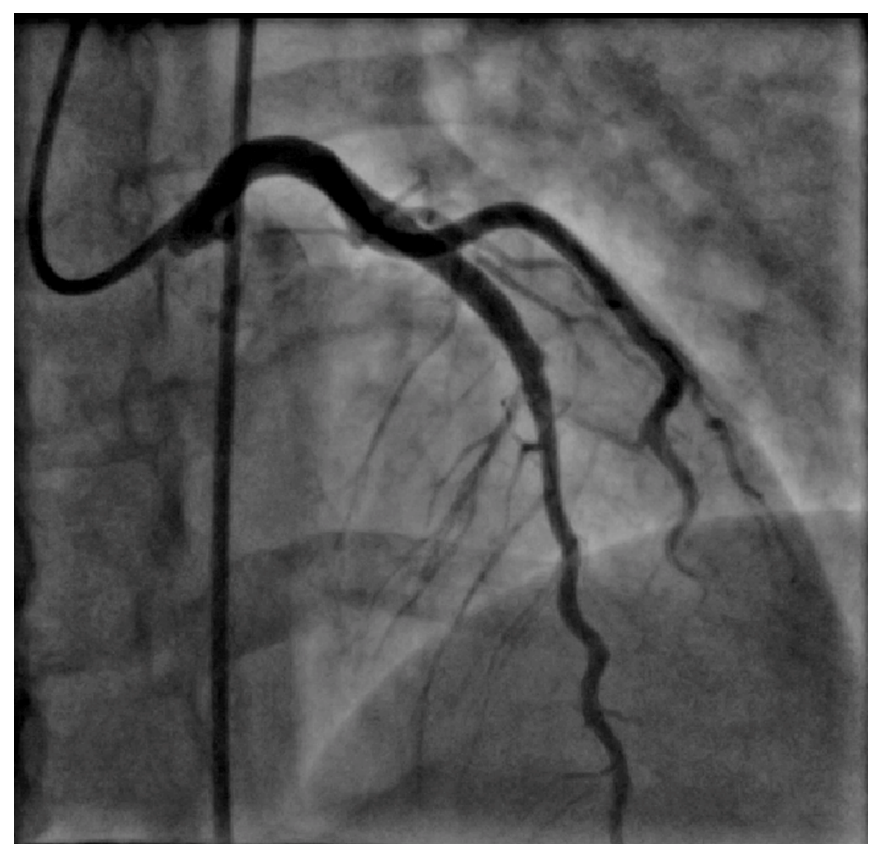

Figure 4. Post-primary PCI angiography revealed TIMI grade III flow at the lesion site.

After about 2 weeks of stent implantation, the patient was reviewed by the cardiologist and the gynecologist. 2D-echocardiography was unremarkable, with improved left ventricular ejection fraction (from $30 \%$ to $45 \%$ ). There were no evidences of thrombosis, residual stenosis, or pericardial effusion. Upon laboratory examination, high-sensitive $\mathrm{C}$ reactive protein (HsCRP) levels were found to be elevated $(7.70 \mathrm{mg} / \mathrm{L}$; reference range $<3$ $\mathrm{mg} / \mathrm{L})$. In addition, total cholesterol (238 mg/dL; reference range: 0 - $200 \mathrm{mg} / \mathrm{dL})$, LDL cholesterol (140 mg/dL; reference range: 0 - $99 \mathrm{mg} / \mathrm{dL}$ ) and triglycerides (215 mg/dL; reference range: $10-170 \mathrm{mg} / \mathrm{dL}$ ) levels were also high. Of significance, the homocysteine levels were within normal range $(7.35 \mu \mathrm{mol} / \mathrm{L}$; reference range: 4.44 $13.56 \mu \mathrm{mol} / \mathrm{L}$ ). The patient was regularly monitored thereafter.

Nearly 4 months after the PCI procedure, an elective caesarean was planned at full-term. Clopidogrel was withheld for a week before caesarean section, while low-dose aspirin was continued. Eventually, the patient delivered a healthy baby (weight: $2.75 \mathrm{~kg}$; APGAR score: 10) without any complications during the caesarean section. Clopidogrel was restarted within 24 hours. After 7 days post-partum, the patient developed slight slurring of speech. Immediate admission under neurologist was sought. Computed tomography scan revealed acute cerebrovascular infarction (Figure 5). The patient was managed appropriately with low-molecular weight heparin and dual antiplatelet therapy. Subsequently, she exhibited a complete recovery. Eventually, she was put on high dose steroids in addition to dual antiplatelet therapy. The patient is doing fine for almost 7 months now. No decision has been taken regarding further pregnancies.

\section{Discussion}

The proportion of primigravida mothers with $\geq 30$ years of age has increased from $4.1 \%$ to $21.2 \%$ over last four decades [4]. With such surge in the maternal age, the incidence of cardiovascular events during pregnancy is likely to increase. This may lead to a progressive increase in demand for both preventive and interventional cardiac care among pregnant women in the coming years [1].

While managing coronary artery disease in pregnant women, coronary catheterization remains underutilized due to concerns related to radiation safety. However, it has been observed that the amount of fetal exposure to radiation during coronary intervention is well below the threshold for teratogenicity at any gestational age [5]. Thus, it may be possible to perform PCI for acute myocardial infarction during pregnancy without any adverse effect on the fetus [1] [6]. In our case, we took additional care to reduce fetal irradiation by limiting the fluoroscopic exposure time and protecting the fetus with lead shields. We believe that coronary catheterization not only helps in differentiating coronary spasm, coronary artery dissection, atherosclerotic disease, or thrombosis, but also 


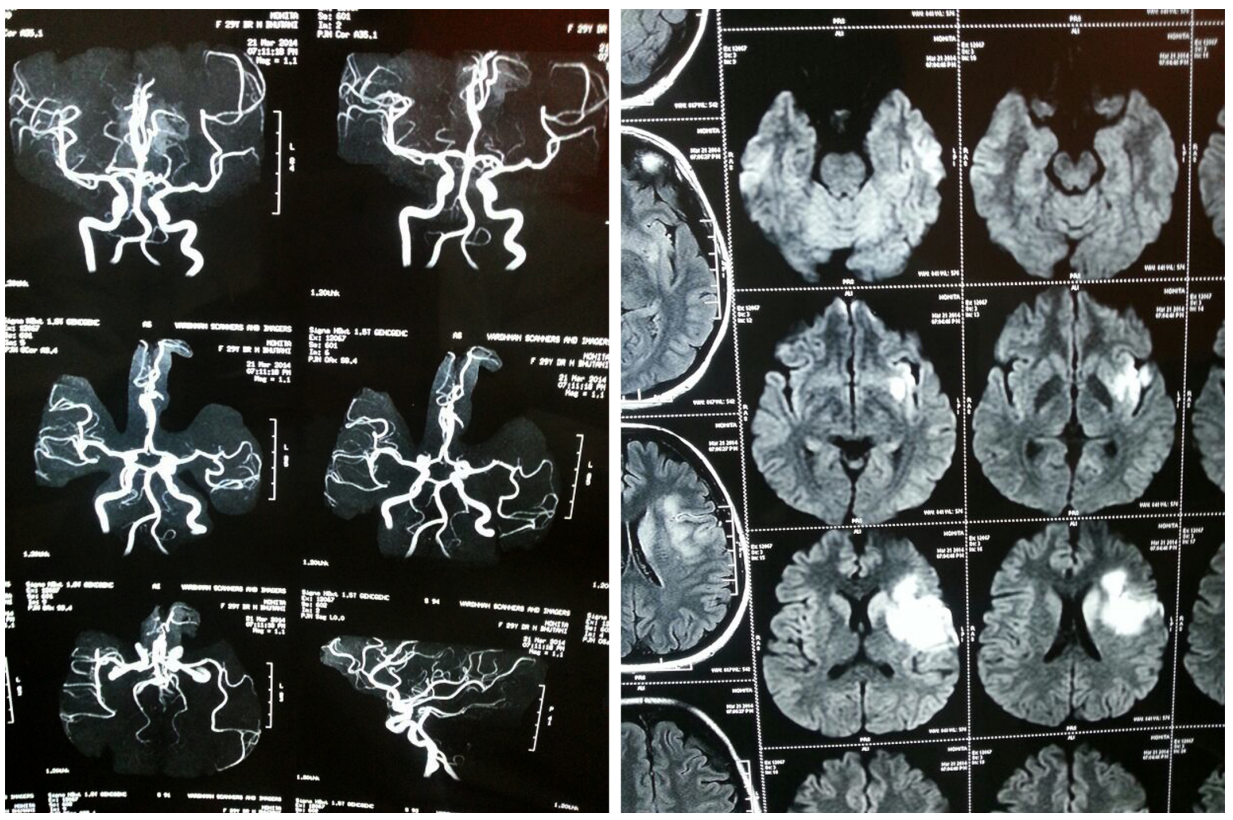

Figure 5. CT scan revealed acute cerebrovascular infarction after 7 days of elective caesarean.

provides a therapeutic tool.

PCI with stenting is a well-documented management modality during pregnancy, and is considered to be safe for maternal and fetal survival [6]. Literature suggests that PCI was preferred over thrombolysis in most such cases [5] [7]. In similar lines, we also do not favor the use thrombolytic therapy during pregnancy as concrete evidences are lacking due to exclusion of pregnant patient in majority of clinical trials in this regard. Considering the relative benefits of drug-eluting stents, we opted for it over bare-metal stent in our patient. We recommended continuation of aspirin and clopidogrel in our patient unless there is a bleeding complication.

Post-primary PCI, it has been recommended to choose the mode of delivery based on clinical and obstetric factors for each patient [5] [6]. Vaginal delivery avoids potential risks associated with anesthesia and a major surgical procedure which include larger blood loss. On the other hand, an elective caesarean section eliminates a long or stressful labor and allows better control of the time of delivery as well as the presence of an appropriate medical team including an experienced obstetrician, obstetric anesthesiologist, cardiologist, and pediatrician [1]. Ultimately, caesarean section was considered as a better alternative in our case.

Earlier, several cases have been reported on successful PCI during pregnancy and eventual delivery of healthy baby [1] [3] [4]. Arimura et al. reported a case of a 43-year-old primiparous woman with acute myocardial infarction, who successfully underwent primary PCI at 21 weeks of gestation and subsequently delivered a healthy baby at 32 weeks gestation upon elective cesarean section [1]. Similarly, Sullebarger et al. described a case of successful PCI in a 38-year-old pregnant woman with non ST elevation myocardial infarction, who delivered a healthy infant at full term [4]. However, reports of complications in the post-partum period after PCI during pregnancy are not reported previously.

The postpartum state is associated with a substantially increased risk of thrombosis and cerebrovascular events, particularly in the first 6 weeks of delivery [8] [9]. In our case, a significant cerebrovascular infarct had occurred within 7 days of delivery. Subsequent to appropriate management, the patient exhibited a complete recovery. Although no particular reason could be identified for the occurrence of cerebrovascular event during the post-partum period in our case, severe theories can be postulated. The prime reason could be irregular use of antiplatelet medications during the last week of pregnancy. Other reasons could be potential use of oral contraceptives before pregnancy and breast feeding in the postpartum period as both these factors could considerably influence the maternal cardiovascular function. In addition, our patient had reported high levels of HsCRP after primary PCI; which is an independent prognostic factor of future coronary events. Hyperhomocysteinemia in the post-partum period is also a powerful risk factor, associated with increased incidence of thrombosis and cardiovascular disease. Although pregnancy itself is not a risk factor, but physiology of pregnancy may also have ex- 
acerbated the underlying myocardial infarction during pregnancy and subsequent cerebrovascular infarction in the post-partum period. This includes increased myocardial and cerebral oxygen demand due to significant increases in blood volume, stroke volume, and heart rate, occurrence of physiological anemia, and profound alterations in the coagulation and fibrinolytic system, leading to increased risk of thrombosis. Advanced maternal age and strong family history of coronary artery disease may also have a role in our case. Identification of possible etiology would guide the prevention and management of such analogous cases in future.

The salient features of the present case could be as follow: 1) Acute myocardial infarction in pregnancy is a very rare occurrence [1]; the present case is one of the very few cases reported from India so far; 2) The patient presented with acute onset of myocardial infarction during the second trimester, while majority of reported cases have depicted that pregnancy-related myocardial infarction is more common during the third-trimester [7]; 3) The symptom-to-door time in our patients was only 20 minutes; 4) our patient lacked conventional coronary risk factors [3] including gestational diabetes or eclampsia, except a family history of coronary artery disease; 5) While the most common cause for pregnancy-related acute myocardial infarction remains atherosclerosis or coronary dissection [7], our case displayed a distinct profile; and 6) An association of acute cerebrovascular event in the post-partum period post-primary PCI has not been reported so far.

Considering the current trend of increase in maternal age and growing incidence of myocardial infarction during pregnancy, it is important that cardiologists remain familiar with the clinical presentation, risk factors, pathophysiology, and potential management options of pregnancy-related myocardial infarction and their interactions with both the pregnant female and the fetus. In addition, evidence-based guidelines should be developed to address appropriate preventative measures as well as acute treatment options for cerebrovascular infarction during pregnancy and in the post-partum period. We also suggest that obstetrics team and neurology team should be consulted to guide in the management of such cases. In addition, an aggressive management approach should be utilized in treating such cases.

\section{Conclusion}

Although rare, acute pregnancy-related myocardial infarction is a devastating event. Management of such patients requires a multidisciplinary approach involving an obstetrician, a cardiologist, and an anesthetist. A careful management in our case allows angina-free pregnancy and delivery after primary PCI in the second trimester. In addition, the occurrence of cerebrovascular infarct in the post-partum period is also managed appropriately. As per the latest follow-up, the patient has remained asymptomatic for past 7 months.

\section{References}

[1] Arimura, T., Mitsutake, R., Miura, S., Nishikawa, H., Kawamura, A. and Saku, K. (2009) Acute Myocardial Infarction Associated with Pregnancy Successfully Treated with Percutaneous Coronary Intervention. Internal Medicine, 48, 1383-1386. http://dx.doi.org/10.2169/internalmedicine.48.2208

[2] Roth, A. and Elkayam, U. (2008) Acute Myocardial Infarction Associated with Pregnancy. Journal of the American College of Cardiology, 52, 171-180. http://dx.doi.org/10.1016/j.jacc.2008.03.049

[3] Cowan, N.C., de Belder, M.A. and Rothman, M.T. (1988) Coronary Angioplasty in Pregnancy. British Heart Journal, 59, 588-592. http://dx.doi.org/10.1136/hrt.59.5.588

[4] Sullebarger, J.T., Fontanet, H.L., Matar, F.A. and Singh, S.S. (2003) Percutaneous Coronary Intervention for Myocardial Infarction during Pregnancy: A New Trend? Journal of Invasive Cardiology, 15, 725-728.

[5] Jaiswal, A., Rashid, M., Balek, M. and Park, C. (2013) Acute Myocardial Infarction during Pregnancy: A Clinical Checkmate. Indian Heart Journal, 65, 464-468. http://dx.doi.org/10.1016/j.ihj.2013.06.016

[6] Bondagji, N.S. (2012) Ischaemic Heart Disease in Pregnancy. Journal of the Saudi Heart Association, 24, 89-97. http://dx.doi.org/10.1016/j.jsha.2011.12.002

[7] Kealey, A. (2010) Coronary Artery Disease and Myocardial Infarction in Pregnancy: A Review of Epidemiology, Diagnosis, and Medical and Surgical Management. Canadian Journal of Cardiology, 26, 185-189. http://dx.doi.org/10.1016/S0828-282X(10)70397-4

[8] Kamel, H., Navi, B.B., Sriram, N., Hovsepian, D.A., Devereux, R.B. and Elkind, M.S. (2014) Risk of a Thrombotic Event after the 6-Week Postpartum Period. New England Journal of Medicine, 370, 1307-1315. http://dx.doi.org/10.1056/NEJMoa1311485

[9] Tate, J. and Bushnell, C. (2011) Pregnancy and Stroke Risk in Women. Womens Health (London, England), 7, 363374. http://dx.doi.org/10.2217/whe.11.19 